Pfizer, AbbVie, Roche, BMS, Novartis, MSD, Saniya Valieva: None declared, Elena Petryaykina: None declared

DOI: 10.1136/annrheumdis-2019-eular.7820

\section{AB0981 10-YEARS EXPERIENCE OF ETANERCEPT USE IN TREATMENT OF JUVENILE IDIOPATHIC ATRTRITIS IN CHELYABINSK REGIONAL PEDIATRIC HOSPITAL}

Galina Glazyrina ${ }^{1,2}$, Olga Sudareva ${ }^{3}{ }^{1}$ South Ural State Medical University, Chelybinsk, Russian Federation; ${ }^{2}$ Chelyabinsk regional pediatric hospital, Chelybinsk, Russian Federation; ${ }^{3}$ Chelyabinsk regional pediatric hospital, Chelyabinsk, Russian Federation

Background: Juvenile idiopathic arthritis (JIA) in recent decades has changed its course, thanks to the use of genetically engineered biological drugs. If therapy with methotrexane is inefficient etanercept is the drug of choice for JIA treatment. Etanercept has been used in Chelyabinsk regional pediatric hospital during 10 years.

Objectives: Performance and safety assessment of etanercept in patients with JIA in Chelyabinsk regional pediatric hospital.

Methods: 51 children aged from 3 to 17 (mean age 10,0 years) diagnosed with JIA were under monitoring (12 boys, 39 girls). Disease duration was from 2 to 15 years (mean duration 5,4 years). JIA was diagnosed based on ILAR diagnostic criteria. Oligo arthritis was diagnosed in 9 children, sero-negative polyarthritis was diagnosed in 34 children. 3 patients had systemic JIA (without active systemic presentations), 5 children had enthesitic JIA. X-ray stage 1-2 was observed in 45 children and stage 3-4 in five. Enhancement antigens HLA B 27 were found in 11 children. In all children methotrexate was ineffective in dose of 15 $\mathrm{mg} / \mathrm{m}^{2}$ during 6-12 months. Etanercept was introduced in dose of $0.8 \mathrm{mg}$ QW. Therapy duration was from 3 months to 8 years (mean duration 29 months). Assessment of disease activity and therapy efficiency was conducted in accordance with ACR pedi criteria. Nonparametric statistical methods were used to compare results.

Results: Prior to etanercept use high disease activity was observed in all children. Mean number of joints with active arthritis was 8 [4, 10] $(\mathrm{Me}[25 ; 75 \%])$. Mean number of joints with functional impairments - 4 [2;10]. Mean ESR (according to Panchenkov) - 23 [10;35] mm/h, CRP $12,0[5,7 ; 32] \mathrm{g} / \mathrm{L}$. Assessment of functional activity according to CHAQ questionnaire - 1,25 [1;2]. Activity assessment according to VAS by doctor - 70 [60;70]. Assessment of parents/patients according to VAS 70 $[60 ; 80]$. No active systemic presentations and eye lesions were found in children under monitoring.

On the background of etanercept therapy a decrease in disease activity was observed in 50 patients. Mean number of joints with active arthritis was $0[0 ; 2](\mathrm{Me}[25 ; 75 \%]) \quad(P \leq 0.0001)$. Mean number of joints with functional impairments - $0[0 ; .2] \quad(P \leq 0.0001)$. Mean $E S R$ was $5[3,6] \mathrm{mm} / \mathrm{h}$ $(P \leq 0.0001), C R P 3[2 ; 4] \mathrm{g} / \mathrm{L}(P \leq 0.0001)$. Assessment of functional activity according to $\mathrm{CHAQ}$ questionnaire was $0,125[0 ; 0,5] \quad(P \leq 0.0001)$. Activity assessment according to VAS by doctor $-10[5 ; 20]$ ( $\mathrm{P} \leq 0.0001)$. Assessment of parents according to VAS $10[5 ; 20](P \leq 0.0001)$.

Clinical disease remission (according to ACR pedi criteria $\geq 90 \%$ ) was observed in 32 patients after 6-12 months of treatment. Remission duration up to now is from 1 to 7 years. Efficiency according to ACR pedi criteria is $70 \%$ in 11 children, $50 \%$ in $5,30 \%$ in 2 .

Etanercept was well-tolerated. Drug was cancelled in 9 patients. 6 patient $(11,8 \%)$ developed bilateral uveitis, one patient had an allergic reaction (rash), one - systemic manifestations, one - urinary tract infection. There were no cases of tuberculosis.

Conclusion: Etanercept therapy was highly effective and safe in patients with JIA. Clinical remission was achieved in $62,7 \%$ children. Decrease in disease activity was observed in $98 \%$ of children. $11,8 \%$ patient developed uveitis, on average, after a year of using etanercept.

\section{REFERENCES}

[1] Lovell D. J., Reiff A., Jones O. Y., Schneider R. et al. Long-term safety and efficacy of etanercept in children with polyarticular-course juvenile rheumatoid arthritis//Arthritis Rheum. 2006; 54: 1987-1994.

Disclosure of Interests: None declared

DOI: 10.1136/annrheumdis-2019-eular.5753

\section{AB0982 \\ CENTRAL NERVOUS SYSTEM ANGIITIS: THE EXPERIENCE OF A TERTIARY PEDIATRIC RHEUMATOLOGY BRAZILIAN CENTER}

Francisco Hugo Rodrigues Gomes ${ }^{1}$, Camila Maria Viana Batista ${ }^{2}$, Jose Savio Menezes Parente ${ }^{2}$, Luana Coelho Benevides ${ }^{1}$, Milena Foizer Leite ${ }^{1}$, Virginia Ferriani ${ }^{1}$, Luciana Martins de Carvalho'. ${ }^{1}$ University of São Paulo, Department of Pediatrics, Division of Pediatric Rheumatology, Ribeirão Preto, Brazil; ${ }^{2}$ University of São Paulo, Department of Pediatrics, Ribeirão Preto, Brazil

Background: Central nervous system (CNS) angiitis is a severe and rare inflammatory brain disease whose course varies from patient to patient. May be associated with infections, malignances, metabolic diseases or systemic collagen vascular disorders. It is classified as primary or idiopathic in the absence of associated systemic diseases. The exact incidence is still undetermined.

Objectives: To describe the clinical and laboratory data, the response to therapy and outcome of patients with CNS angiitis seen at a Tertiary Pediatric Rheumatology Center.

Methods: This is a retrospective, single-center chart review study of pediatric patients with clinical diagnoses of CNS angiitis, followed up in a Tertiary Pediatric Rheumatology Center, from January 2009 to December 2018. Diagnosis was confirmed by magnetic resonance angiography (MRA) or after exhaustive exclusion of other causes.

Results: Nine patients ( 4 girls) were enrolled in this study. Mean age at inclusion and at diagnosis was 11.5 years $(7.8$ to 19.9$)$ and 8 years $(3.5$ to 12,5$)$, respectively. Among the 9 patients, $4(44 \%)$ were diagnosed with primary CNS angiitis. Polyarteritis nodosa $(n=4)$ and Behçet disease $(n=1)$ were the aetiologies of secondary CNS angiitis. Main clinical features were sudden onset of seizures $(67 \%)$ and headache $(55 \%)$. Other important symptoms were: decreased level of consciousness, hemiparesis and neurocognitive dysfunction. In the cerebral spinal fluid, protein was elevated in $22 \%$ of patients. The pattern of lesions was bilateral in $67 \%$ and multifocal in 55\%, being suggestive of ischemic lesions by CNS magnetic resonance in $78 \%$ of the cases. The MRA was conclusive in all cases of secondary and normal in only one case of primary CNS angiitis. Increased erythrocyte sedimentation rate, C-reactive protein and leukocyte count were more frequently observed in patients with secondary CNS angiitis as compared to patients with primary angiitis. In one case of primary CNS angiitis with negative vascular study, the Von Willebrand factor antigen was positive, being useful in the differential diagnosis. Steroids were administered in $100 \%$ of patients, associated with immunosuppressants in $7 / 9$ cases. Induction therapy with intravenous cyclophosphamide was used in $78 \%$ of the cases and in the maintenance phase, azathioprine in $78 \%$ of them. No relapses occurred. The most commonly clinical sequel observed was residual epilepsy (55\%).

Conclusion: In this study, seizures were the most frequently symptom found. Steroids plus immunosuppressants were effective in the disease control. MRA was positive in all cases of secondary CNS angiitis, being effective in the diagnosis of this condition. Recognition of findings and adequate diagnosis guides the treatment, which should be specific to the underlying cause, aiming to provide a good neurologic outcome.

\section{REFERENCES}

[1] TWILT M, BENSELER S. Central Nervous System vasculitis in adults and children. Handbook of Clinical Neurology, 2016;133: 283-300.

[2] DEMIR S, SAG E, DEDEOGLU F, et al. Vasculitis in Systemic Autoinflammatory Diseases. Frontiers in Pediatrics 2018; 6:377.

Disclosure of Interests: None declared

DOI: 10.1136/annrheumdis-2019-eular.7446

\section{AB0983 PENTRAXIN 3 AS A MARKER OF DISEASE ACTIVITY IN JUVENILE IDIOPATHIC ARTHRITIS PATIENTS}

Waleed Hassan ${ }^{1}$, Eman Behiry ${ }^{2}$, Shorouk Abdelshafy ${ }^{3} .{ }^{1}$ Benha University, Rheumatology and Rehabilitation, Benha, Egypt, ${ }^{2}$ Benha University, Clinical and Chemical Pathology, Benha, Egypt, ${ }^{3}$ Benha University, Diagnostic Radiology, Benha, Egypt

Background: Pentraxin-3 (PTX3) is a secretory acute phase protein which is produced and expressed in many immune cells especially macrophages, fibroblasts and endothelial cells at different inflammatory sites [1]. 
PTX3 is known to have an interesting role in regulation of innate immunity and it is a key player in regulation of many inflammatory reactions [2]

Objectives: This study aimed to measure serum and synovial fluid (SF) levels of PTX3 in juvenile idiopathic arthritis (JIA) patients and to correlate them with different clinical, laboratory and musculoskeletal ultrasound parameters of disease activity.

Methods: We measured PTX3 in the serum $(n=57)$ and SF samples $(n=18)$ from 57 JIA patients and in the serum from twenty healthy control. Disease activity was calculated using the Juvenile Arthritis Disease Activity Score in 27 joints (JDAS27) and musculoskeletal ultrasound examination (MSUS) was performed using grey scale (GS) and power Doppler (PD) 10-joint score (bilateral knee, ankle, wrist, elbow and the $2^{\text {nd }}$ metacarpophalangeal (MCP) joints)[3]; Erythrocyte sedimentation rate (ESR), C-reactive protein (CRP) levels, serum ferritin, rheumatoid factor (RF) titre were measured and the Juvenile arthritis multidimensional assessment report (JAMAR) was documented.

Results: Serum PTX3 level was significantly higher in JIA patients $(3.59 \pm$ $2.38 \mathrm{ng} / \mathrm{mL})$ compared to serum level in the healthy controls $(1.5 \pm 0.9$ $\mathrm{ng} / \mathrm{mL}) \quad(\mathrm{p}<0.001)$. There was no significant difference between SF PTX3 level $(4.73 \pm 2.62 \mathrm{ng} / \mathrm{mL})$ and its level in paired serum samples(4.23 \pm $2.93 \mathrm{ng} / \mathrm{mL})(p=0.59)$. JIA patients with systemic onset subtype $(n=13)$ had higher PTX3 serum levels $(5.57 \pm 2.52 \mathrm{ng} / \mathrm{mL})$ compared to serum level in the oligoartiular $(n=25)$ and polyarticular $(n=19)$ subtypes $(2.88 \pm$ $2.13 \mathrm{ng} / \mathrm{mL}$ and $3.18 \pm 11.92 \mathrm{ng} / \mathrm{mL}$ respectively) ( $\mathrm{p}=0.001$ and 0.005 respectively). In JIA patients, serum PTX3 level significantly correlated with JDAS27 $(r=0.52, p<0.05)$, CRP titres $(r=0.46, p<0.05)$ and serum ferritin $(r=0.48, p<0.05)$. Both serum and SF PTX3 levels significantly correlated with PD score $(r=0.51$ and 0.48 respectively, $p<0.05)$. SF PTX3 ( $=0.001$ ) was shown to be superior to serum PTX3(p=0.02), ESR ( $p$ $=0.07)$ and comparable to CRP $(p=0.001)$ at predicting $\mathrm{PD}$ synovitis score.

Conclusion: JIA patients have significantly increased serum and synovial fluid levels of Pentraxin 3 that remarkably correlated with the JDAS27 and MSUS parameters of inflammations suggesting that it could be a useful marker to reflect JIA disease activity.

\section{REFERENCES}

[1] Liu S, Qu X, Liu F, Wang C. Pentraxin 3 as a Prognostic Biomarker in Patients with Systemic Inflammation or Infection. Mediators Inflamm; 2014:421-429.

[2] Mantovani A, Garlanda C, Doni A, Bottazzi B. Pentraxins in innate immunity: from C-reactive protein to the long pentraxin PTX3. J Clin Immunol. 2008 Jan;28(1):1-13

[3] Collado P, Naredo E, Calvo C, Gamir ML, Calvo I, García ML, et al. Reduced joint assessment vs comprehensive assessment for ultrasound detection of synovitis in juvenile idiopathic arthritis. Rheumatology (Oxford). 2013 Aug; 52(8):1477-84.

Disclosure of Interests: None declared

DOI: 10.1136/annrheumdis-2019-eular.5780

\section{AB0984 INTERSTITIAL LUNG DISEASE IN CHINESE JIA PATIENTS}

Assunta Ho, Kate Chan, LI Albert. Prince of Wales Hospital, The Chinese University of Hong Kong, Paediatrics, Hong Kong, Hong Kong (SAR)

Background: Rheumatic diseases and or medications (for example methotrexate) used to treat the underlying condition are associated with the development of interstitial lung disease (ILD). Development of ILD in subjects with juvenile idiopathic arthritis (JIA) is assumed to be rare, however, severe respiratory complications are increasingly reported in JIA patients.

Objectives: To describe the prevalence of respiratory diagnoses and ILD in a group of Chinese JIA patients at a tertiary center.

Methods: Retrospective chart review of JIA patients attending rheumatology clinic during 2012 to 2019

Results: Fifty-eight cases of JIA were identified. Thirty-three (57\%) received DMARDs, majority were on methotrexate (MTx, 24, 73\%) and sulphasalazine (SSZ, 14, 42\%). Eleven (19\%) were currently or previously on biologics (adalimumab 5, etanercept 5, anakinra 1, previous exposure: infliximab 1, etanercept and anakinra 1).

Respiratory diagnosis was made in 11 (19\%) patients. There were 2 $(3.4 \%)$ cases of ILD diagnosed during the study period (see below). The rest were asthmatics. Among them, 9 were on DMARDs [MTx $8(89 \%)$ SSZ 4(44\%), leflunomide 2(LEF), hydroxychloroquine 1(HCQ)] or biologics [Etanercept 3(33\%), adalimumab 1, tocilizumab and anakinra 1(sequential use)]. Four subjects were on combination DMARDs [MTx\&SSZ in 2, 1 each for $M T x+L E F+H C Q+S S Z$ (sequential combination)\& MTx+LEF]. Three received combination DMARDs and biologics (1 each for MTX+adalimuumab, MTx+etanercept \& MTx+anakinra)

Cases of ILD

One was a SJIA patient diagnosed at 2 years old. She failed methotrex ate, etanercept \& tocilizumab (infusion reaction). She was on low dose prednisolone, methotrexate and anakinra when she presented to us with acute right heart failure requiring ICU care 3 years after diagnosis. Advanced clubbing was noted (fig 1). HRCT thorax on presentation showed severe interstitial lung disease with marked fibrosis and traction bronchiectasis (fig 2). She was diagnosed acute on chronic cor pulmonale and pulmonary hypertension secondary to ILD.

The other was an oligoarticular JIA patient, diagnosed at 2 years old. She was refractory to intra-articular steroid injections and combination DMARDs including MTX, SSZ, LEF \& HCQ. Arthritis was controlled by Etanercept. At 12 years of age, she started to have chest pain. Lung function test showed restrictive lung pattern. HRCT thorax showed pulmonary fibrosis.

Conclusion: From this Chinese cohort we identified 2 cases of ILD. They did not present with any symptoms until advanced stage or when complications set in. Both were very young at diagnosis and were difficult to control, as evidenced by the use of multiple DMARDs and biologics including methotrexate, IL6 and IL1 blockade. The causes of ILD are believed to be multifactorial. For severe case, prognosis is poor ${ }^{1}$ Although rare, methotrexate induced pneumonitis do occur.

Symptoms of ILD may not be obvious until late stage. Clinical examination alone is not sensitive enough to detect disease progression. Asthma is common, as in this cohort, and may further complicated the assessment. It is recommended in the recent SHARE initiative on JSLE to screen for lung disease ${ }^{2}$. We believe it should be performed in JIA patients as well. 6 minute walk test is a noninvasive way to assess cardiopulmonary functional status and is validated in children ${ }^{3}$. Patients with unexplained chest symptoms or impaired lung function should be referred to pulmonologist for further assessment.

\section{REFERENCES}

[1] Kimura Y, et al. Pulmonary Hypertension and other potentially fatal pulmonary complications in systemic juvenile idiopathic arthritis. Arthritis Care Res 2013;65(5):856-52

[2] Groot N, et al. European evidence-based recommendations for diagnosis and treatment of childhood-onset systemic lupus erythematosus: the SHARE initiative. Ann Rheum Dis 2017;76:1788-96

[3] $\mathrm{Li} \mathrm{AM}$, et al. The six-minute walk test in healthy children: reliability and valdity. Eur Respir J 2005;25:1057-60

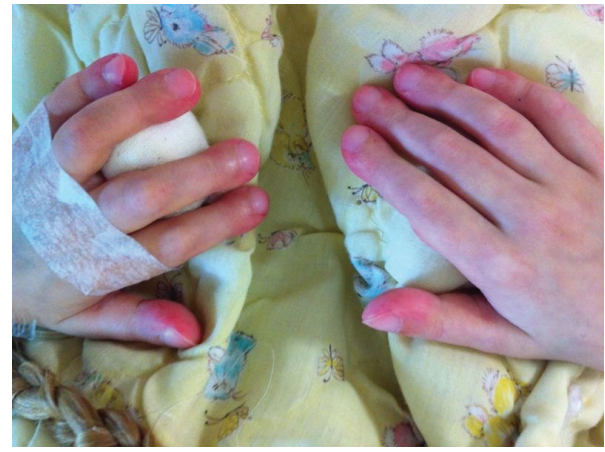

Figure 1 